\title{
RELAXED STATES OF A MAGNETIZED PLASMA WITH MINIMUM DISSIPATION
}

\author{
B. Dasgupta*, P. Dasgupta**, \\ M. S. Janaki*, T. Watanabe ${ }^{* * *}$ and T. Sato*** \\ *Saha Institute of Nuclear Physics \\ I/AF, Bidhannagar, Calcutta 700 064, India \\ **Department of Physics, University of Kalyani, Kalyani 741235, India \\ $* * *$ Theory and Computer Simulation Center \\ National Institute for Fusion Science, Toki, Gifu 509-52, Japan
}

\begin{abstract}
.
Relaxed state of a slightly resistive and turbulent magnetized plasma is obtained by invoking the Principle of Minimum Dissipation which leads to

$$
\nabla \times \nabla \times \nabla \times \mathbf{B}=\Lambda \mathbf{B}
$$

A solution of the above equation is accomplished using the analytic continuation of the Chandrasekhar-Kendall eigenfunctions in the complex domain. The new features of this theory is to show (i) a single fluid can relax to an MHD equilibrium which can support pressure gradient even without a longterm coupling between mechanical flow and magnetic field (ii) field reversal (RFP) in states that are not force-free.
\end{abstract}


In the well-known theory of relaxation of magnetoplasma, Taylor [1] proposed that the process of relaxation is governed by the principle of minimum total magnetic energy and invariance of total (global) magnetic helicity $K=\int_{V} \mathbf{A} \cdot \mathbf{B} d V$ where the integration is over the entire volume, the latter being the most significant invariant in the theory of relaxation. Accordingly the relaxed state of a magnetoplasma satisfies the corresponding Euler-Lagrange equation

$$
\nabla \times \mathbf{B}=\lambda \mathbf{B}
$$

with constant $\lambda$, and, consequently, is a force-free state. Taylor's theory is quite successful in explaining a number of experimental results, including those of RFP. However, relaxed states as envisaged by Taylor, have only zero pressure gradient.

Extensive numerical works by Sato and his collaborators have established [2], [3] the existence of self-organized states with finite pressure, i.e. these states are governed by the magnetohydrodynamic force balance relation, namely, $\mathbf{j} \times \mathbf{B}=\nabla p$, rather than $\mathbf{j} \times \mathbf{B}=0$. Recently, it has been

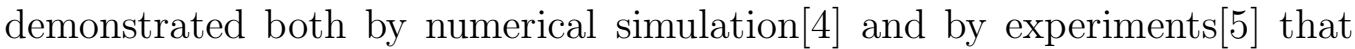
counter-helicity merging of two spheromaks can produce a Field-Reversed Configuration (FRC). The FRC has zero toroidal magnetic field and the plasma is confined entirely by poloidal magnetic field. It has a finite pressure with a relatively high value of $\beta$. It may be concluded that $\mathrm{FRC}$, with its non zero perpendicular component of current, is a relaxed state and it is a distinctly non-force free state. From the point of view of plasma relaxation, the formation of FRC through the counter-helicity merging of two spheromaks is a unique process where a non-force free state emerges from the fusion of two Taylor states. The conclusion is that there exists a general class of relaxed states which are not always force-free, and Taylor's force-free states constitute only a subclass of this wider class. While Taylor states do not support any pressure gradient, equilibrium obtained from the principle of minimum energy accommodates pressure gradients only in presence of flow. Several attempts [6], [8] have been made in the past to obtain relaxed states which could support finite pressure gradient, a large number of them making use of the coupling of the flow with magnetic field [9]-[12].

The principle of "minimum rate of entropy production", formulated by Prigogine 13] and others, is believed to play a major role in many problems of irreversible thermodynamics. Dissipation, along with nonlinearity, is ubiquitous in systems which evolve towards self-organized states. Another closely related concept, the principle of minimum dissipation rate was used for the first time by Montgomery and Phillips 14 in an MHD problem to understand the steady state profiles of RFP configuration under the constraint of constant rate of supply and dissipation of helicity the usual physical boundary 
conditions for a conducting wall. It may be pointed out that the principle of minimum dissipation was also discussed by Chandrasekhar and Woltzer 16 in a sequel to the complete general solution of the force-free equation by Chandrasekhar and Kendall [15]. The minimum dissipation rate hypothesis was later used by a number of authors [17, 18 to predict the current and magnetic field profiles of driven dissipative systems.

This paper deals with the question of determining the field configurations assumed by a magnetofluid in a relaxed state in absence of any external fields, while maintaining that the relaxation is governed by the hypothesis of minimum rate of energy dissipation. It is our conjecture that relaxed states could be characterized as the states of minimum dissipation rather than states of minimum energy. The novel feature of our work is to show that it is possible for a single fluid to relax to an MHD equilibrium with a magnetic field configuration which can support pressure gradient, even without a longterm coupling between the flow and the magnetic field. In a recent work, Steinhauer 12 has claimed that single fluid MHD theory can admit only a force free state and one need to take recourse to a two fluid theory so that electromechanical coupling produces pressure gradient and a non force free state. Our work establishes that none of these requirements need be satisfied to obtain a relaxed state of the desired kind.

In what follows we derive the Euler-Lagrange equation from a variational principle with minimum energy dissipation and conservation of total magnetic helicity, solve the equation in terms of the analytically continued Chandrasekhar-Kendall eigenfunctions, discuss the important role played by the boundary conditions, and present our results for the flux, field reversal parameter and pinch parameter. We also compute the helicity integral, and show the plots of magnetic field, current, and pressure profiles. The field reversal parameter from our theory is definitely in better agreement with the experimental value than what is obtained from Taylor's theory.

We consider a closed system of an incompressible, resistive magnetofluid, without any mean flow velocity, described by the standard MHD equations in presence of a small but finite resistivity $\eta$. In the absence of any externally imposed electric fields, the ohmic dissipation rate $R$ is itself a time varying quantity. However, it is possible to find constraints that are better preserved than the rate of energy dissipation, so that the system self-organizes to certain relaxed states, which remain stable over time scales short compared to ohmic dissipation time. In this case, helicity still serves to hold as a good constraint as it decays at a time scale much slower in comparison to the decay time scale of the rate of energy dissipation as is evident from the simulation works of Zhu et. al. [3]. In the following, we compare the decay rates of the energy 
dissipation rate and helicity. The former is obtained as

$$
\frac{d R}{d t}=2 \frac{\eta^{2}}{S^{2}} \sum_{k} k^{4} \mathbf{b}_{k}^{4}
$$

while the latter turns out to be

$$
\frac{d K}{d t}=-2 \frac{\eta}{S} \sum_{k} k \mathbf{b}_{k}^{2} .
$$

From the above two equations, we see that the decay rate of energy dissipation is once again $\mathrm{O}(1)$ at scale lengths for which $k \approx S^{\frac{1}{2}}$. But at these scale lengths, helicity dissipation is only $\mathrm{O}\left(S^{-1 / 2}\right)<<1$. Thus, we may expect that in presence of small scale turbulence, the rate of energy dissipation decays at a faster rate than helicity.

We therefore minimize the ohmic dissipation $R=\int \eta \mathbf{j}^{2} d V$ subject to the constraints of helicity $\int \mathbf{A} \cdot \mathbf{B} d V$. The variational equation is given by

$$
\delta \int\left(\eta \mathbf{j}^{2}+\bar{\lambda} \mathbf{A} \cdot \mathbf{B}\right) d V=0
$$

where $\bar{\lambda}$ is Lagrange's undetermined multiplier. The variation can be shown to lead to the Euler-Lagrange equation

$$
\nabla \times \nabla \times \nabla \times \mathbf{B}=\Lambda \mathbf{B}
$$

where, $\Lambda=\bar{\lambda} / \eta$ is a constant. The surface terms in the equation vanish if we consider the boundary condition $\delta \mathbf{A} \times \mathbf{n}=$ as well as $\mathbf{j} \times \mathbf{n}=0$, which is the physical boundary condition we will impose in the problem.

We like to emphasize that eq.(3) is a general equation which embraces the Woltzer-Taylor equation ( i.e. eq. (1) ) as a special case. Now we proceed to construct a solution of eq.(3) and show that the general solutions can have $\mathbf{j} \times \mathbf{B} \neq 0$. In other words, eq.(3) may lead to a non force-free state.

The solution of eq.(3) can be constructed using the Chandrasekhar-Kendall (CK) eigenfunctions. Chandrasekhar and Kendall's solution[15] of the equation $\nabla \times \mathbf{B}=\lambda \mathbf{B}$ can be written (with three parameters $\mu, m, k$ in cylindrical coordinates), as,

$$
\mathbf{B}(\mu, m, k)=\lambda \nabla \Phi \times \nabla z+\nabla \times(\nabla \Phi \times \nabla z)
$$

where, $\Phi=J_{m}(\mu r) \exp [i(m \theta-k z)]$ with $\lambda^{2}=\mu^{2}+k^{2}$. Here, $J_{m}$ is a Bessel Function of order $m$ and the value of $\mu$ in the argument is determined from the boundary condition at $r=a$, which is given as $(\hat{\mathbf{n}} \cdot \mathbf{B})_{r=a}=0$

Analytic continuation of the above solution for complex values of $\mu$ (or $k$ ) is straightforward. For real values of $\lambda$ (and hence of $\mu$ and $k$ ) the operator 
$(\nabla \times)$ has been proved to be self-adjoint, but not so in the larger space spanned by the analytically continued CK solutions.

We introduce the complex parameters

$$
\mu_{n}=\left[\left(\mu^{2}+k^{2}\right) \exp (4 n \pi i / 3)-k^{2}\right]^{1 / 2}, n=1,2
$$

so that $\mu_{n}^{2}+k^{2}=\lambda^{2} \omega^{2 n}, \omega=\exp (2 \pi i / 3)$, and define

$$
\begin{aligned}
& \mathbf{B}_{\mathbf{1}}=\mathbf{B}(\mu, m, k)=\lambda \nabla \Phi \times \nabla z+\nabla \times(\nabla \Phi \times \nabla z) \\
& \mathbf{B}_{\mathbf{2}}=\mathbf{B}\left(\mu_{1}, m, k\right)=\lambda \omega \nabla \Phi_{1} \times \nabla z+\nabla \times\left(\nabla \Phi_{1} \times \nabla z\right) \\
& \mathbf{B}_{\mathbf{3}}=\mathbf{B}\left(\mu_{2}, m, k\right)=\lambda \omega^{2} \nabla \Phi_{2} \times \nabla z+\nabla \times\left(\nabla \Phi_{2} \times \nabla z\right)
\end{aligned}
$$

In the last two expressions above, $\Phi_{1}$ and $\Phi_{2}$ are obtained from $\Phi$ by replacing $\mu$ by $\mu_{1}$ and $\mu_{2}$ respectively.

A solution of eq.(3) can now be obtained as a linear combination of $\mathrm{B}_{1}, \mathrm{~B}_{2}, \mathrm{~B}_{3}$ :

$$
\mathbf{B}=\alpha_{1} \mathbf{B}_{\mathbf{1}}+\alpha_{2} \mathbf{B}_{\mathbf{2}}+\alpha_{3} \mathbf{B}_{\mathbf{3}}
$$

where $\alpha_{i}$ are constants, with at least two of them non-zero. It can be easily demonstrated that the expression for $\mathbf{B}$ given in (7) is a solution of eq.(3) with $\Lambda=\lambda^{3}$.

A reasonable boundary condition is to assume a perfectly conducting wall, so that

$$
\mathbf{B} \cdot \mathbf{n}=0, \quad \mathbf{j} \times \mathbf{n}=0 \quad \text { at } \quad r=a
$$

The boundary conditions given by eq. (8) suffice to fix the arbitrary constants

$$
\begin{aligned}
\frac{\alpha_{2}}{\alpha_{1}} & =-\frac{\left.\omega^{2}\left(B_{1 \theta} B_{2 z}^{*}-B_{2 \theta}^{*} B_{1 z}\right)\right|_{r=a}}{\left.\left(B_{2 \theta} B_{2 z}^{*}-B_{2 \theta}^{*} B_{2 z}\right)\right|_{r=a}} \\
\alpha_{3} & =\alpha_{2}^{*}
\end{aligned}
$$

The magnetic fields at the boundary $r=a$ have to obey the following relation for non-trivial values of the constants $\alpha_{i}$

$$
2 B_{1 r} \operatorname{Im}\left(B_{2 \theta} B_{2 z}^{*}\right)-2 B_{1 \theta} \operatorname{Im}\left(\omega^{2} B_{2 r} B_{2 z}^{*}\right)+2 B_{1 z} \operatorname{Im}\left(\omega^{2} B_{2 r} B_{2 \theta}^{*}\right)=0
$$

From eq. (6), it is evident that $B_{2}$ and $B_{3}$ are complex conjugate of one another. This, together with the relations obtained in eq. (10), shows that the magnetic field given by eq. (7) is a real field. We also list the following expressions for the $m=0, k=0$ state (cylindrically symmetric state) 
obtained from eqs. (4)-(7) :

$$
\begin{aligned}
& B_{r}=0 \\
& B_{\theta}=\lambda^{2} \alpha_{1}\left[J_{1}(\lambda r)+2 \operatorname{Re}\left(\frac{\alpha_{2}}{\alpha_{1}} \omega^{2} J_{1}(\lambda \omega r)\right)\right] \\
& B_{z}=\lambda^{2} \alpha_{1}\left[J_{0}(\lambda r)+2 \operatorname{Re}\left(\frac{\alpha_{2}}{\alpha_{1}} \omega^{2} J_{0}(\lambda \omega r)\right)\right]
\end{aligned}
$$

For a given value of $m$ and $k a$, the value of $\lambda a$ can be obtained from the boundary condition given by eq. (11). It is to be noted that for the cylindrically symmetric state the boundary condition is trivially satisfied and hence does not determine $\lambda a$. It can be easily proved that the state of minimum dissipation is equivalent to the state of minimum value of $\Lambda$. To get the numerical value of $\lambda$ for $m \neq 0$, we solve numerically eq.(11) and obtain $\lambda a=3.11$ and $k a=1.23$ as the minimum values of $\lambda a$ and $k a$ for the $m=1$ state.

The only undetermined constant in eq. (7) is the value of $\alpha_{1}$ (the value of the field amplitude) which can be determined by specifying the toroidal flux $\Phi_{z}$. The $m=k=0$ state is responsible for non-zero values of toroidal flux which is obtained as

$$
\Phi_{z}=2 \pi \alpha_{1} \lambda a\left[J_{1}(\lambda a)+2 \operatorname{Re}\left[\frac{\alpha_{2}}{\alpha_{1}} \omega J_{1}(\lambda \omega a)\right]\right]
$$

A couple of dimensionless quantities that have proved useful in describing laboratory experiments are the field reversal parameter $F=B_{z}(a) /<B_{z}>$ and the pinch parameter $\Theta=B_{\theta}(a) /<B_{z}>$, where $<. .>$ represents a volume average. After substituting the expressions for $B_{z}(a)$ etc, we get

$$
\begin{aligned}
F & =\frac{\lambda a}{2} \frac{J_{0}(\lambda a)+2 \operatorname{Re}\left[\left(\alpha_{2} / \alpha_{1}\right) \omega^{2} J_{0}(\lambda \omega a)\right]}{J_{1}(\lambda a)+2 \operatorname{Re}\left[\left(\alpha_{2} / \alpha_{1}\right) \omega J_{1}(\lambda \omega a)\right]} \\
\Theta & =\frac{\lambda a}{2} \frac{J_{1}(\lambda a)+2 \operatorname{Re}\left[\left(\alpha_{2} / \alpha_{1}\right) \omega^{2} J_{1}(\lambda \omega a)\right]}{J_{1}(\lambda a)+2 \operatorname{Re}\left[\left(\alpha_{2} / \alpha_{1}\right) \omega J_{1}(\lambda \omega a)\right]}
\end{aligned}
$$

The pinch ratio $\Theta$ is related to the ratio of the current and flux and is a physically controllable quantity. For the Taylor state $\Theta=\lambda a / 2$.

The details of any relaxed state are determined by two physically meaningful parameters, the toroidal flux and the volts-seconds of the discharge. The toroidal flux as defined earlier serves to determine the field amplitude and the volts-seconds describes the helicity of the relaxed state through the relation: volts $-\mathrm{sec}=$ helicity $/ \mathrm{flux}^{2}$. We therefore calculate the helicity integral (global helicity) from our solution for the $m=0, k=0$ state and 
get

$$
\begin{aligned}
K & =4 \pi^{2} \alpha_{1}^{2} \frac{R}{a}(\lambda a)^{3}\left[J_{0}^{2}(\lambda a)+J_{1}^{2}(\lambda a)-\frac{2}{\lambda a} J_{0}(\lambda a) J_{1}(\lambda a)\right] \\
& +2 R e\left[\frac{\alpha_{2}^{2}}{\alpha_{1}^{2}}\left[J_{0}^{2}(\lambda \omega a)+J_{1}^{2}(\lambda \omega a)-\frac{2}{\lambda \omega a} J_{0}(\lambda \omega a) J_{1}(\lambda \omega a)\right]\right] \\
& +2 R e\left[\frac{\alpha_{2}}{\alpha_{1}} \frac{2}{\lambda a\left(1-\omega^{2}\right)}\left[J_{0}(\lambda a) J_{1}(\lambda \omega a)-\omega J_{1}(\lambda a) J_{0}(\lambda \omega a)\right]\right] \\
& +2 \frac{\left|\alpha_{2}\right|^{2}}{\alpha_{1}^{2}} \frac{1}{\lambda a(1-\omega)}\left[\omega J_{0}\left(\lambda \omega^{2} a\right) J_{1}(\lambda \omega a)-J_{1}\left(\lambda \omega^{2} a\right) J_{0}(\lambda \omega a)\right]
\end{aligned}
$$

We then calculate the volts-seconds for the cylindrically symmetric state using $\lambda a=3.11$. For the minimum value of $\lambda a=3.11$, the critical value of volts-seconds $=12.8 \mathrm{R} / \mathrm{a}$. For values of volts-seconds less than this critical value, a lower value of $\lambda a$ is obtained from solving the equation for $K / \Phi_{z}^{2}$ so that the cylindically symmetric state is the relaxed state for minimum energy dissipation. For values of volts-seconds greater than the critical value the system relaxes to the helically distorted state with $\lambda a=3.11$ which is obtained as a mixture of the $m=0, k=0$ and the $m=1, k \neq 0$ states as in the case of Taylor's theory.

The profiles for the current and magnetic field are shown in Figs. 1 and 2 for the $m=0, k=0$ state with the value of $\lambda a<3.11$ (i.e., volts-sec $<12.8 R / a)$. At a value of $\lambda a$ greater than 2.95 , the magnetic field profile $B_{z}$ vs $r$ shows a reversal near the edge ( Fig.2 ). Also, $j_{z}$ and $j_{\theta}$ go to zero at the wall because of the boundary conditions we have chosen. The values of both $F$ and $\Theta$ at the boundary $r=a$ are evaluated and $F$ is plotted against pinch ratio $\Theta$ ( Fig.3 ). It is observed that $F$ reverses at a value of $\Theta=2.4$, $(\lambda a=2.95)$ whereas for the Taylor state the reversal is achieved at $\Theta=1.2$. However, this field reversed state supports pressure gradient in constrast to the Taylor state. The q-profile, where $q=r B_{z} / R B_{\theta}$ is shown in Fig. 4 for $\lambda a=3.0$.

The pressure profile can be obtained from the relation $\mathbf{j} \times \mathbf{B}=\nabla p$. For the $m=0, k=0$ state, the only nonvanishing component of the pressure gradient exists in the radial direction. The pressure profile is shown in Fig. 5 for the $m=k=0$ state with $\lambda a=3.0$ which is the minimum energy dissipation, field reversed state.

To conclude, the principle of minimum dissipation is utilized together with the constraints of constant magnetic helicity to determine the relaxed states of a magnetoplasma not driven externally. The variational principle leads to a remarkable Euler-Lagrange equation, and it is shown that this equation involving higher order curl operator can be solved in terms of of an analytical continuation of Chandrasekhar-Kendall functions in the complex 
domain with appropriate boundary conditions. This relaxed state obtained from single fluid MHD supports pressure gradient. A coupling between magnetic field and flow is not an essential criterion for having a non-zero pressure gradient. Further, it is shown that a non force-free state with field reversal properties can exist.

One of the authors (BD) wishes to acknowledge the kind hospitality of ICTP, Trieste, Italy, where a part of the work has been carried out during his visit as an Associate. The author gratefully acknowledges many illuminating and inspiring discussions with Predhiman Kaw, David C. Montgomery, Swadesh Mahajan and Zensho Yoshida. 


\section{References}

[1] J. B. Taylor, Phys. Rev. Lett., 33, 139, (1974)

[2] T. Sato, H. Takamaru, Phys. Plasmas 2, 3609 (1995), T. Sato and the Complexity Sim. group, Phys. Plasmas 3, 2135 (1996), S. Zhu, R. Horiuchi, T. Sato and the Complexity Sim. group, Phys. Plasmas 3, 2821 (1996).

[3] S. Zhu, R. Horiuchi and T. Sato, Phys. Rev. E. 51, 6047 (1995).

[4] B. Dasgupta, T. Sato, T. Hayashi, K. Watanabe and T. Watanabe, Trans. Fusion Tech. 27, 374, (1995). T. Watanabe, B. Dasgupta, T. Sato, T. Hayashi and K. Watanabe, (1995) Int. Sherwood Fus. Theo. Conf., April 3-5, Nevada, USA,

[5] Y. Ono et al Phys. Fluids, B 5, 3691, (1993).

[6] E. Hameiri and J. Hammer, Phys. Fluids, 25, 1855, (1982).

[7] E. Minardi, Plasma Phys. Contr. Fusion, 31,229, (1989).

[8] K. Avinash, Phys. Fluids B 5, 3857 (1992).

[9] J. M. Finn and T. M. Antonsen, Phys. Fluids, 26, 3540, (1983)

[10] R.N. Sudan, Phys. Rev. Lett. 42, 1278, (1979).

[11] R. Horiuchi and T. Sato, Phys. Fluids, 31, 1143, 1988.

[12] L. C. Steinhauer and A. Ishida, Phys. Rev. Lett. 79, 3423, (1997)

[13] P. Glansdorff and I. Prigogine, Thermodynamic Theory of Structure, Stability and Fluctuations, Wiley Interscience, New York, (1971)

[14] D. Montgomery and L. Phillips, Phys. Rev. A. 38, 2953, (1988)

[15] S. Chandrasekhar and P. C. Kendall, Astro. Phys. J., 126, 457, (1957)

[16] S. Chandrasekhar and L. Woltzer, Astro. Phys. J., 127, , (1958)

[17] C.Y. Wang and A. Bhattacharjee, Phys. Fluids 3, 3462, (1991).

[18] M.K. Bevir, A. Caloutsis and C. G. Gimblett, Plasma Phys. Contr. Fusion, 34133 (1993). 


\section{Figure Captions}

Fig.1 $j_{z}(r)$ vs $r$ for the axisymmetric state $m=0, k=0$, and $\lambda a=3.0$. The current vanishes at the edge because of the boundary condition $\mathbf{j} \times \mathbf{n}=0$.

Fig.2 Magnetic field profile for the axisymmetric state with $\lambda a=3.0$, showing field reversal near the edge.

Fig.3 The field reversal parameter $F$ against the pinch parameter $\Theta$, the field reversal occuring at $\Theta=2.4$. The dotted curve represents the plot for the minimum energy state of Taylor.

Fig.4 The q-profile for the axisymmetric state.

Fig. 5 The pressure profile $p$ vs $r$ for $\lambda a=3.0$. 


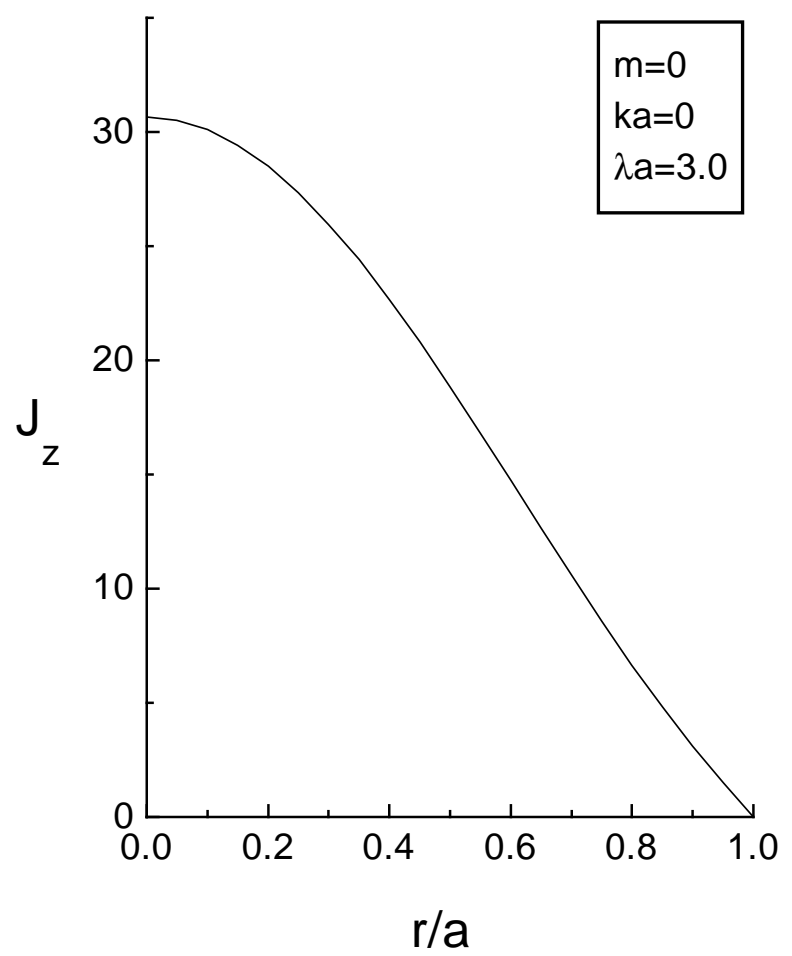




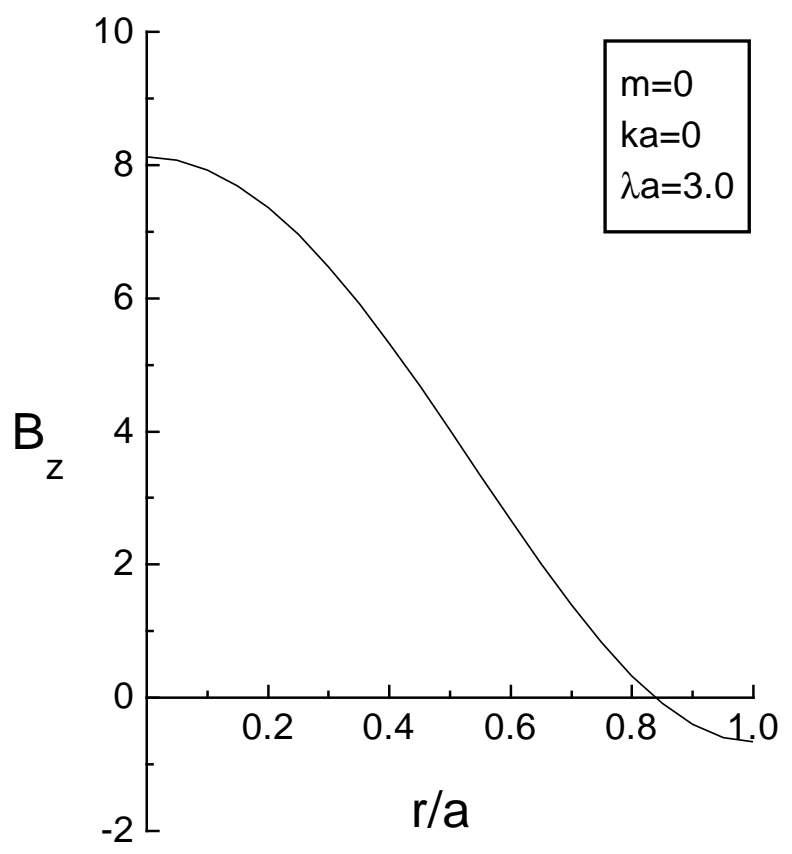




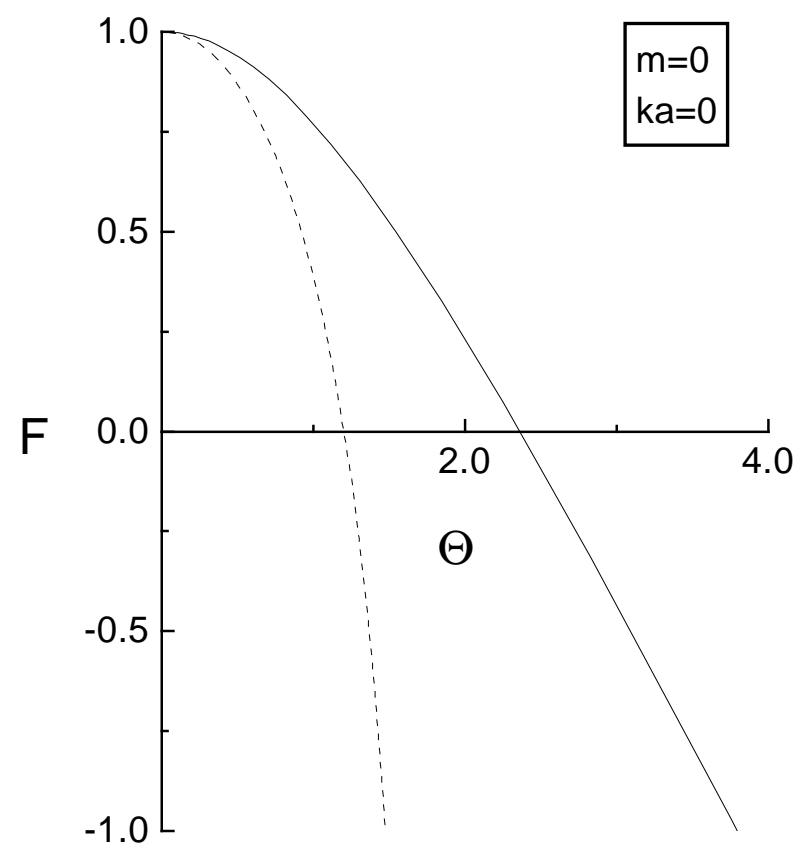




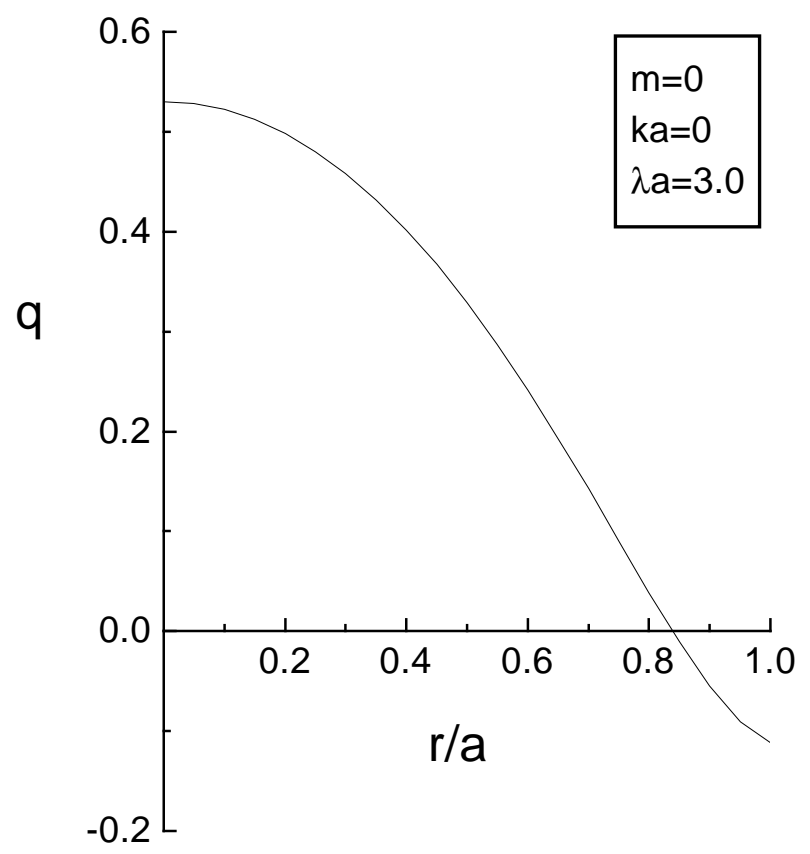




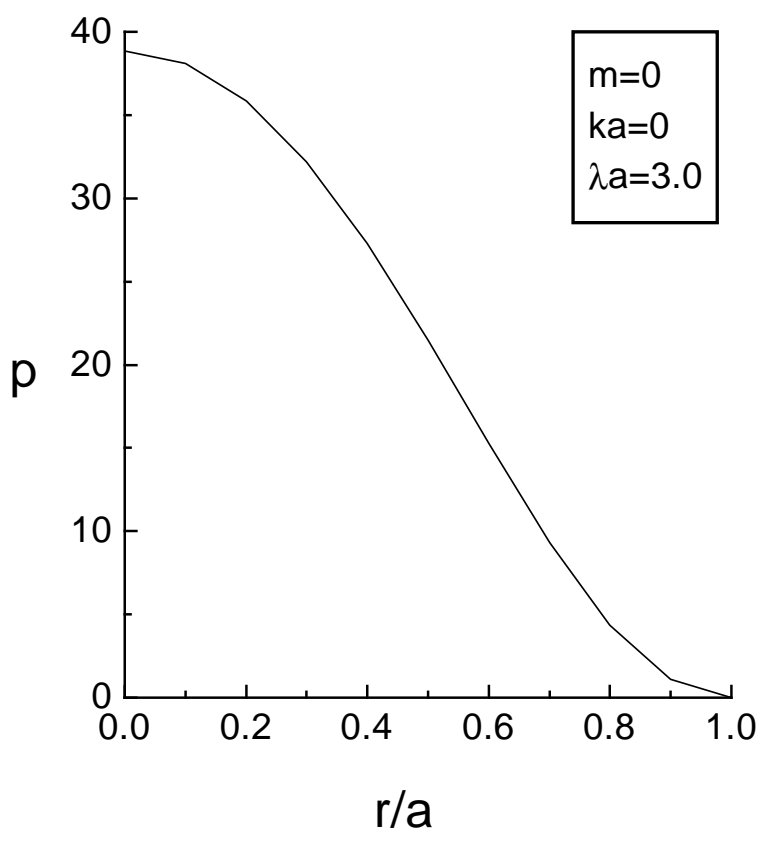

